\title{
Análise da prevalência da Síndrome de von Hippel-Lindau em pacientes portadores de hemangioblastomas do Sistema Nervoso Central
}

Analysis of the prevalence of von Hippel-Lindau Syndrome in patients with hemangioblastomas of the Central Nervous System

Análisis de la prevalencia del Síndrome de von Hippel-Lindau en pacientes con hemangioblastomas del Sistema Nervioso Central

Recebido: 21/01/2022 | Revisado: 24/01/2022 | Aceito: 29/01/2022 | Publicado: 03/02/2022

Gustavo Soares Gomes Barros Fonseca ORCID: https://orcid.org/0000-0002-7287-1409

Universidade CEUMA, Brasil

E-mail: gustavosgbf@gmail.com

Vivianne Maria Laranjeiras Monte Freire ORCID: https://orcid.org/0000-0002-0364-9724 Universidade CEUMA, Brasil

E-mail: vivianelaranjeiiras@ hotmail.com Lais Lima Bonfim ORCID: https://orcid.org/0000-0002-2816-3464 Universidade CEUMA, Brasil

E-mail: laisbonfim@hotmail.com

Elana Cristina da Silva Penha ORCID: https://orcid.org/0000-0002-1034-6967

Universidade UNIFAMAZ, Brasil

E-mail: elanaa_penha@hotmail.com

João Victor Araújo Guimarães ORCID: https://orcid.org/0000-0003-2842-4510 Universidade CEUMA, Brasil

E-mail: joaoviictorguimaraes98@hotmail.com

Raphael Erick Lima Pereira ORCID: https://orcid.org/0000-0002-8721-986X Universidade CEUMA, Brasil

E-mail: raphaerick@hotmail.com

Gabriel de Sousa Macedo ORCID: https://orcid.org/0000-0003-3445-0379 Universidade CEUMA, Brasil

E-mail: gdesousa01110@gmail.com

Filipe Augusto Lopes Cajubá de Britto ORCID: https://orcid.org/0000-0002-7687-6186 Universidade CEUMA, Brasil

E-mail: filipecajjuba@outlook.com

Letícia Muniz de Abreu Murad ORCID: https://orcid.org/0000-0002-9000-8852 Universidade CEUMA, Brasil E-mail: iicemurad@gmail.com

Alessandra do Rosário Brito ORCID: https://orcid.org/0000-0002-2703-4476 Universidade UNIFAMAZ, Brasil E-mail: alessaww@yahoo.com.br

Lara Letícia Teixeira Reis ORCID: https://orcid.org/0000-0002-6052-9091 Universidade CEUMA, Brasil

E-mail: laraleticireis1@gmail.com

Amanda Cordeiro Santos ORCID: https://orcid.org/0000-0001-6118-5983 Universidade CEUMA, Brasil

E-mail: amandacordeiiros14@gmail.com Agostinho Rodrigues Mesquita Neto ORCID: https://orcid.org/0000-0003-0259-3420 Universidade UNIFAMAZ, Brasil 
Julianne Souza Prazeres

ORCID: https://orcid.org/0000-0002-3174-5422

Universidade CEUMA, Brasi

E-mail: juliannesouuza_@hotmail.com

Luiza Mariana Batista Lima Cunha

ORCID: https://orcid.org/0000-0003-4736-1092 Universidade CEUMA, Brasil

E-mail: luizamarianaliima@gmail.com

Ana Vitória Feitosa Barroso Maia

ORCID: https://orcid.org/0000-0002-8561-0646 Universidade CEUMA, Brasil

E-mail: vicfeiitosa@hotmail.com

Carolynne Weba da Silva

ORCID: https://orcid.org/0000-0001-8426-6794 Universidade CEUMA, Brasil

E-mail: carolwebdasilva@gmail.com

Fernando de Abreu Borges

ORCID: https://orcid.org/0000-0002-0127-0155 Universidade CEUMA, Brasil

E-mail: fernandoaborges@gmail.com

Monaliza Brito de Almeida

ORCID: https://orcid.org/0000-0002-3376-3501 Universidade FACID, Brasil

E-mail: monalizabrito@gmail.com

Samantha Cunha Vieira

ORCID: https://orcid.org/0000-0002-8831-2855 Universidade CEUMA, Brasil

E-mail: samanthacviieira@gmail.com

Isabela Coelho Brito Soares

ORCID: https://orcid.org/0000-0001-7697-4571 Universidade FACID, Brasil

E-mail: isabelacbriito@hotmail.com

Liciana Tapety Sá

ORCID: https://orcid.org/0000-0003-3006-7938 Universidade CEUMA, Brasil

E-mail: licianatapetys@gmail.com

Lucca Adriano Pereira

ORCID: https://orcid.org/0000-0002-1431-337X Universidade CEUMA, Brasil E-mail: luccap99@gmail.com

Thalita Martins Bezerra

ORCID: https://orcid.org/0000-0002-6229-1312 Universidade CEUMA, Brasil

E-mail: thalita_martins124@hotmail.com

Ivana Mota Soares

ORCID: https://orcid.org/0000-0002-9064-3717 Universidade CEUMA, Brasil

E-mail: ivanamotaa@gmail.com

Rebeca Machado Castro Sousa

ORCID: https://orcid.org/0000-0003-4680-5181 Universidade CEUMA, Brasil

E-mail: becaccastro31@hortmail.com

Maria Beatriz Celedonio Coelho

ORCID: https://orcid.org/0000-0001-7168-6562 Universidade CEUMA, Brasil

E-mail: mariabeatriizcoelho@ hotmail.com

Felipe Ramos Caldeira

ORCID: https://orcid.org/0000-0002-7468-5191 Universidade UNIRG, Brasil

E-mail: santos_med@yahoo.com

\section{Resumo}

Hemangioblastomas (HBs) são neoplasias benignas comumente localizadas no cerebelo e medula espinhal e podem surgir de forma esporádica ou relacionados à Síndrome de von Hippel-Lindau (VHL). Quando há associação com a VHL, tumores multifocais podem surgir, diferentemente dos esporádicos, que são isolados. A VHL é uma doença hereditária relacionada ao desenvolvimento de tumores benignos e malignos, sendo os mais comuns os hemangioblastomas do Sistema Nervoso Central e os angiomas de retina. A doença é causada pela ineficácia do gene supressor de tumor VHL (pVHL) em um alelo no cromossomo 3p25-26. A presença da VHL em pacientes com HBs leva o paciente a um pior prognóstico, por facilitar a metastatização. Dessa forma, o objetivo da seguinte revisão é analisar a prevalência da Síndrome de von Hippel-Lindau em pacientes portadores de hemangioblastomas do Sistema 
Nervoso Central (SNC). Trata-se de uma revisão bibliográfica sistemática que utilizou as plataformas PubMed, SciELO e Google Scholar como bases de dados para pesquisa de artigos científicos, com recorte temporal entre 2016 e 2022 , na língua inglesa. De acordo com o mecanismo de busca, 4 resultados foram encontrados após os critérios de exclusão nas bases de dados PubMed e Google Scholar. Constatou-se, portanto, que há moderada prevalência da VHL em pacientes portadores de HBs do SNC. Dentre os estudos, a menor taxa de prevalência da VHL em pacientes com HBs foi de $23 \%$, enquanto a maior taxa foi de aproximadamente $60 \%$, com uma média geral de $\pm 39,2 \%$, ao serem considerados todos os estudos que envolveram esses pacientes.

Palavras-chave: Hemangioblastoma; Síndrome de von Hippel-Lindau; Prevalência.

\begin{abstract}
Hemangioblastomas (HBs) are benign neoplasms commonly located in the cerebellum and spinal cord and may arise sporadically or related to von Hippel-Lindau Syndrome (VHL). When there is an association with VHL, multifocal tumors may arise, unlike sporadic tumors, which are isolated. VHL is a hereditary disease related to the development of benign and malignant tumors, the most common being central nervous system hemangioblastomas and retinal angiomas. The disease is caused by the inefficacy of the Tumor Suppressor Gene VHL (pVHL) in an allolo on chromosome 3p25-26. The presence of VHL in patients with HBs leads the patient to a worse prognosis, as it facilitates metastatization. Thus, the aim of the following review is to analyze the prevalence of von Hippel-Lindau syndrome in patients with central nervous system (CNS) hemangioblastomas. This is a systematic bibliographic review that used the PubMed, SciELO and Google Scholar platforms as databases for research of scientific articles, with a time frame between 2016 and 2022, in English. According to the search engine, 4 results were found after the exclusion criteria in the PubMed and Google Scholar databases. It was found, therefore, that there is a moderate prevalence of HLV in patients with CNS HBs. Among the studies, the lowest prevalence rate of HIV in patients with HBs was $23 \%$, while the highest rate was approximately $60 \%$, with an overall average of $\pm 39.2 \%$, when considering all studies involving these patients.
\end{abstract}

Keywords: Hemangioblastoma; Von Hippel-Lindau syndrome; Prevalence.

\title{
Resumen
}

Los hemangioblastomas (HB) son neoplasias benignas comúnmente localizadas en el cerebelo y la médula espinal y pueden surgir esporádicamente o relacionadas con el síndrome de von Hippel-Lindau (VHL). Cuando hay una asociación con VHL, pueden surgir tumores multifocales, a diferencia de los tumores esporádicos, que se aíslan. La VHL es una enfermedad hereditaria relacionada con el desarrollo de tumores benignos y malignos, siendo los más comunes los hemangioblastomas del sistema nervioso central y los angiomas retinianos. La enfermedad es causada por la ineficacia del gen supresor de tumores VHL (pVHL) en un alolo en el cromosoma 3p25-26. La presencia de VHL en pacientes con HB lleva al paciente a un peor pronóstico, ya que facilita la metastatización. Por lo tanto, el objetivo de la siguiente revisión es analizar la prevalencia del síndrome de von Hippel-Lindau en pacientes con hemangioblastomas del sistema nervioso central (SNC). Se trata de una revisión bibliográfica sistemática que utilizó las plataformas PubMed, SciELO y Google Scholar como bases de datos para la investigación de artículos científicos, con un marco temporal entre 2016 y 2022, en inglés. Según el buscador, se encontraron 4 resultados tras los criterios de exclusión en las bases de datos PubMed y Google Scholar. Se encontró, por lo tanto, que existe una prevalencia moderada de HLV en pacientes con HB del SNC. Entre los estudios, la tasa de prevalencia más baja de HIV en pacientes con HB fue del $23 \%$, mientras que la tasa más alta fue de aproximadamente el $60 \%$, con un promedio general de $\pm 39,2 \%$, al considerar todos los estudios con estos pacientes.

Palabras clave: Hemangioblastoma; Síndrome de von Hippel-Lindau; Prevalencia.

\section{Introdução}

Hemangioblastomas (HBs) são neoplasias vasculares benignas que surgem mais comumente na fossa posterior, na região do cerebelo e da medula espinhal e podem surgir esporadicamente ou relacionados à Síndrome de von Hippel-Lindau (VHL) (Patel et al., 2019; Maher et al., 2011; Wanebo et al., 2003). Quando associados à VHL, podem-se surgir tumores multifocais, diferentemente dos esporádicos, os quais são geralmente isolados. Geralmente ocorrem em adultos jovens e em pacientes de meia-idade, com leve predileção masculina em adultos, com relação M:F de 2:1 (Malleo, 2010; Cervio et al., 2015; Guerrero-Galindo et al., 2012; Kanno et al., 2014).

A Síndrome de von Hippel-Lindau é uma patologia hereditária com incidência de 1/36.000 nascidos vivos que consiste em um desenvolvimento de tumores benignos e malignos, sendo os mais comuns os hemangioblastomas do Sistema Nervoso Central e os angiomas de retina (Lonser at al., 2003; Nordstrom-O’Brien et al., 2010; Findeis-Hosey et al., 2016). Ademais, carcinomas de células renais de células claras, feocromocitomas e tumores neuroendócrinos do pâncreas fazem parte do pool da 
VHL (Chittiboina \& Lonser, 2015; Seizinger et al., 1988; Frantzen et al., 2012; Sampaio et al., 2022).

A doença é causada pela perda germinativa da função do gene supressor de tumor VHL (pVHL) em um alelo no cromossomo 3p25-26. Assim, um evento somático de "segundo golpe" leva à perda do outro alelo e, consequentemente, no desenvolvimento tumoral. A perda celular da função do VHL aumenta a expressão e estabilização do fator induzido por hipóxia (HIP), o qual facilita a tumorigênese de hemangioblastomas (Chittiboina \& Lonser, 2015; Seizinger et al., 1988; Frantzen et al., 2012).

A presença da VHL em pacientes portadores de HBs leva o paciente a um pior prognóstico, pois há maiores chances de metastatização através de um mecanismo angiogênico, recidiva tumoral e desenvolvimento de tumores multifocais, o que não ocorre nos esporádicos. Dessa forma, é necessário que haja uma detecção precoce de HBs nesses pacientes, por meio de um exame neurológico bem realizado por parte do profissional, além de exames complementares, como a ressonância magnética, além de um manejo agressivo, a fim de erradicar os focos neoplásicos por meio da neurocirurgia (Koo et al., 2016; Pavesi et al., 2008; Ammerman et al., 2006).

Dessa forma, nosso objetivo é analisar a prevalência da Síndrome de von Hippel-Lindau em pacientes portadores de hemangioblastomas do Sistema Nervoso Central.

\section{Metodologia}

Trata-se de uma revisão bibliográfica sistemática, de natureza quantitativa, que utilizou as plataformas PubMed, Scientific Eletronic Library On-line (SciELO) e Google Scholar como base de dados para pesquisa dos artigos científicos. Foram utilizadas literaturas publicadas com recorte temporal de 2016 a 2022, na língua inglesa, que abordavam sobre a prevalência da Síndrome de von Hippel-Lindau em pacientes portadores de hemangioblastomas do Sistema Nervoso Central.

Os descritores utilizados seguiram a descrição dos termos DeCs (Descritores em Saúde) e Medical Subject Headings (MeSH) no idioma inglês, como mostra o Quadro 1.

Quadro 1 - Estratégia de busca para o estudo.

(“Hemangioblastoma”[All Fields]) OR ("hemangioblastoma"[All Fields]) OR ("hemangioblastoma”[MeSH Terms])) AND (“von hippel lindau disease"[All Fields])) OR (“von hippel lindau syndrome"[MeSH Terms])) AND ("prevalence”[All Fields])

Fonte: Autores (2022).

Nesta revisão, os critérios de exclusão utilizados foram: Livros, documentos de projetos de dissertação, resumos em eventos, editoriais, revisões de literatura, relatos de caso isolados, artigos que não cumpriam os critérios de inclusão e artigos duplicados, conforme o Fluxograma 1. 
Fluxograma 1 - Sistematização da filtragem de artigos para confecção do estudo.

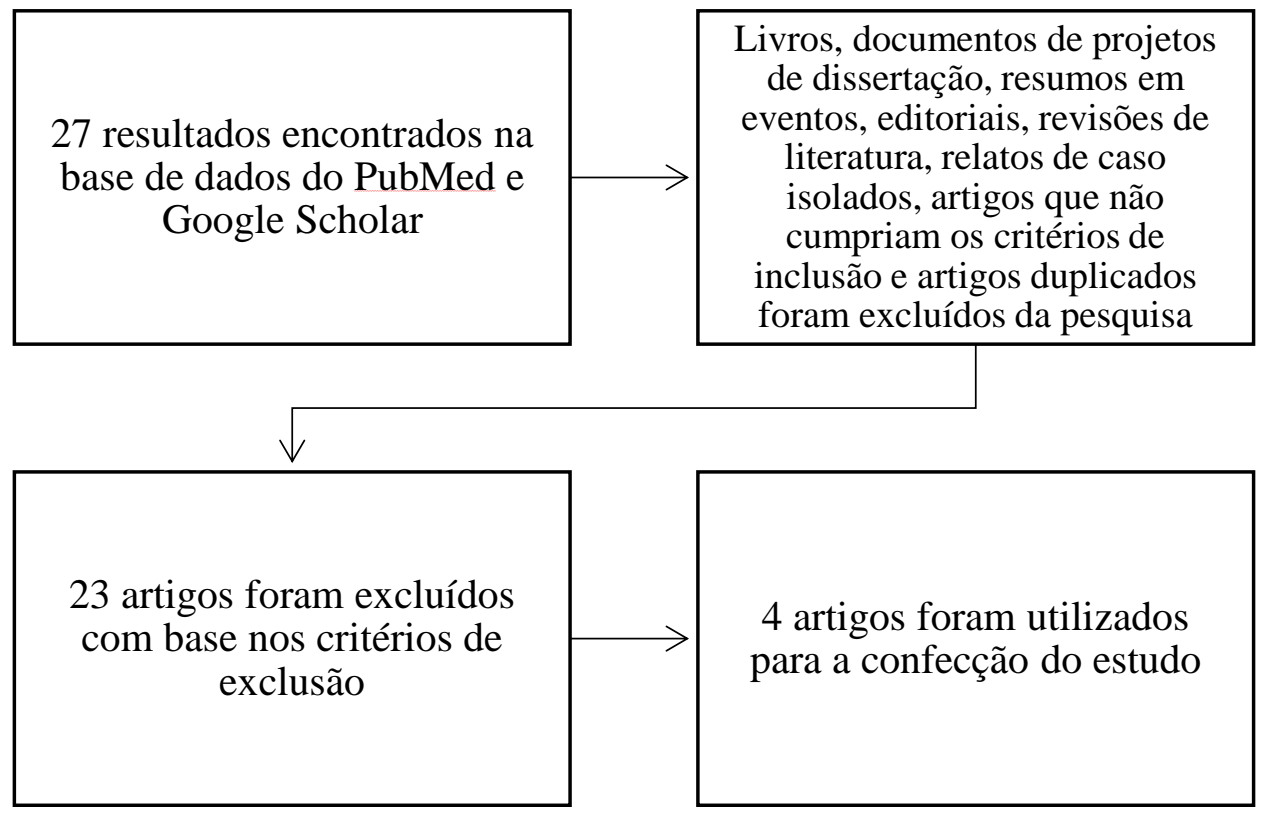

Fonte: Autores (2022)

\section{Resultados e Discussão}

A escolha dos artigos a serem utilizados nesta revisão foi realizada por meio da leitura do título, resumo e, por fim, da leitura do artigo na íntegra, sendo realizada uma análise criteriosa dos artigos fundamentados nos critérios de inclusão e exclusão supracitados.

De acordo com o mecanismo de busca, 27 foram encontrados resultados, sendo eles na base de dados PubMed e Google Scholar, com 0 na base SciELO. Dos 27 resultados obtidos, 23 foram excluídos com base nos critérios de exclusão para a pesquisa e, além disso, não abordavam de forma objetiva sobre o tema, sendo apenas 4 artigos utilizados na confecção do estudo. Todos os artigos escolhidos serão expostos no Quadro 2, seguindo a ordem de ano de sua publicação. 
Quadro 2 - Artigos selecionados nas bases de dados PubMed e Google Scholar.

\begin{tabular}{|c|c|c|}
\hline TÍTULO & AUTOR, ANO & OBSERVAÇÕES \\
\hline $\begin{array}{l}\text { Hemangioblastomas with } \\
\text { leptomeningeal dissemination: Case } \\
\text { series and review of the literature }\end{array}$ & Koo et al. (2016) & $\begin{array}{l}\text { O estudo incluiu } 23 \text { pacientes portadores de hemangioblastomas, sendo } 12 \\
\text { do sexo feminino e } 11 \text { do sexo masculino, com idades de } 4 \text { a } 59 \text { anos, com } \\
\text { uma média de idade de } \pm 38,1 \text { anos. A prevalência de pacientes com a } \\
\text { Síndrome de von Hippel-Lindau foi de } 39 \% \text { ( } 7 / 23 \text { ), sendo quatro do sexo } \\
\text { feminino e três do sexo masculino. Dos } 7 \text { pacientes, } 6 \text { apresentavam } \\
\text { hemangioblastomas de manifestação cerebelar, e } 1 \text { na medula espinhal. }\end{array}$ \\
\hline $\begin{array}{l}\text { Incidence and Prognosis of Spinal } \\
\text { Hemangioblastoma: A Surveillance } \\
\text { Epidemiology and End Results } \\
\text { Study }\end{array}$ & $\begin{array}{l}\text { Westwick et al. } \\
\text { (2016) }\end{array}$ & $\begin{array}{l}\text { Em } 133 \text { casos, o hemangioblastoma foi o décimo tipo de tumor espinhal } \\
\text { intradural mais comum, representando } 2,1 \% \text { ( } 133 \text { de } 6.156) \text { de todos os } \\
\text { tumores espinhais. Em relação à presença do gene supressor de tumor Von } \\
\text { Hippel-Lindau (pVHL), foi observado que houve predomínio em } \\
\text { aproximadamente } 60 \% \text { dos pacientes. }\end{array}$ \\
\hline $\begin{array}{l}\text { Clinical Features and Surgical } \\
\text { Outcomes in Patients with } \\
\text { Cerebellopontine Angle } \\
\text { Hemangioblastomas: Retrospective } \\
\text { Series of } 23 \text { Cases }\end{array}$ & Cheng et al. (2017) & $\begin{array}{l}\text { O estudo incluiu } 23 \text { pacientes, sendo } 14 \text { homens e } 9 \text { mulheres, } \\
\text { apresentaram hemangioblastomas no ângulo pontinocerebelar e } 34,8 \% \\
(8 / 23) \text { tinham Síndrome de von Hippel-Lindau (VHL). A ressecção total } \\
\text { bruta foi alcançada em } 22 \text { pacientes ( } 95,6 \%) \text {. O seguimento médio foi de } \\
45,1 \pm 36,2 \text { meses (intervalo de } 3 \text { a } 144 \text { meses). }\end{array}$ \\
\hline $\begin{array}{c}\text { Central nervous system } \\
\text { hemangioblastomas: An age- } \\
\text { stratified analysis }\end{array}$ & Wang et al. (2020) & $\begin{array}{l}\text { O estudo analisou } 187 \text { pacientes portadores de hemangioblastomas na } \\
\text { medula espinhal e cerebelo, sendo } 102 \text { homens e } 85 \text { mulheres. Foi } \\
\text { observado que } 23 \% \text { ( } 43 / 187) \text { dos pacientes foram diagnosticados com } \\
\text { VHL. } 47,6 \% \text { dos pacientes com idade entre } 0 \text { e } 20 \text { anos, } 23 \% \text { dos pacientes } \\
\text { com idade entre } 21 \text { e } 59 \text { anos e } 6,4 \% \text { dos pacientes com idade } \geq 60 \text { anos } \\
\text { foram diagnosticados com VHL. Pacientes idosos obtiveram um risco } \\
\text { significativamente menor de VHL e menor taxa de recorrência tumoral. } \\
\text { Tumor sólido, Síndrome de von Hippel-Lindau e localização no tronco } \\
\text { encefálico foram associados a um mau prognóstico em pacientes com } \\
\text { hemangioblastomas. }\end{array}$ \\
\hline
\end{tabular}

Fonte: Autores (2022).

Os hemangioblastomas (HBs) do Sistema Nervoso Central (SNC) são tumores benignos e de lento crescimento, os quais possuem ou não associação com a Síndrome de von Hippel-Lindau (VHL). Os pacientes portadores de HBs que não possuem o gene supressor de tumor VHL (pVHL) adquiriram os tumores esporadicamente, sendo estes aproximadamente 25\% (Koo et al., 2016; Westwick et al., 2016; Cheng et al., 2017; Wang et al., 2020). Dessa forma, nota-se a grande prevalência da Síndrome nos pacientes com HBs do SNC.

A Síndrome de von Hippel-Lindau é uma síndrome hereditária neurocutânea neoplásica autossômica dominante que afeta vários órgãos, como o pâncreas, rins, medula e cerebelo. Assim sendo, podem surgir tumores benignos e malignos em múltiplos órgãos, sendo os mais comuns os hemangioblastomas cerebelares e os angiomas de retina. O gene supressor de tumor VHL (pVHL) está localizado no braço curto do cromossomo 3 (3p25.3) (Cheng et al., 2017).

No estudo descrito por Koo et al. (2016), dos 23 pacientes portadores de HBs do SNC, apenas 7 apresentaram o pVHL específico para a síndrome, com uma prevalência de 39\% e média de idade de $\pm 38,1$ anos. Desses 7 pacientes, 6 apresentaram o hemangioblastoma no cerebelo e apenas 1 na medula espinhal. Notou-se que a recidiva tumoral pós-ressecção cirúrgica foi similar nos pacientes com HBs esporádicos e associados à VHL. Isso pode ser explicado pelo mecanismo semelhante de disseminação do tumor através dos fatores de crescimento relativos à angiogênese. Sob outra perspectiva, foi visto que os HBs relacionados à Síndrome de von Hippel-Lindau (HBs-VHL), embora histologicamente similares aos esporádicos, possuem um desenvolvimento mais agressivo e com maior potencial de disseminação e velocidade de crescimento de lesões multifocais e podem ter um forte efeito negativo nos resultados do paciente a longo prazo. 
Além disso, Koo et al. (2016) observaram que a causa mais comum de morte da maior parte dos pacientes foi devido à insuficiência respiratória, por compressão pontomedular ou do cordão cervical. Uma detecção precoce e manejo agressivo pode melhorar significamente o prognóstico dos pacientes portadores dos HBs-VHL.

Já Westwick et al. (2016), em seu estudo, demonstraram que, dos 6.156 casos dos tumores espinhais intradurais, o hemangioblastoma foi o décimo mais comum, representando 2,1\% (133 pacientes). Foi notado que, dos 133 casos de HBs, 53 encontravam-se na faixa etária de 15-44 anos (39,8\%), sendo ela a de maior incidência quando comparada aos extremos de idade ( $\mathrm{p}=0,01370$ e IC 95\% = 0,10-0,017), o que é significantemente estatístico para afirmar que há maior incidência em pacientes jovens e adultos.

Por outro lado, Westwick et al. (2016) observaram que a raça branca influencia na aparição dos HBs, visto que essa faz parte de 79,7\% dos 133 casos (106 pacientes), sendo $\mathrm{p}=0,015$ e IC 95\% = 0,012-0,018). Ademais, foi observado que houve predomínio em mais da metade dos pacientes a presença do gene supressor de tumor Von Hippel-Lindau (pVHL), responsável pela manifestação da Síndrome de von Hippel-Lindau. Assim, nota-se a grande prevalência de HBs-VHL nos pacientes do estudo em questão.

Segundo Cheng et al. (2017), foram incluídos 23 pacientes, sendo 14 do sexo masculino e 9 do sexo feminino, com HBs localizados no ângulo pontinocerebelar. Foi visto que pacientes com hemangioblastomas císticos apresentaram melhor prognóstico neurológico $(\mathrm{p}=0,035)$ em comparação com aqueles portadores de tumores sólidos. Além disso, 34,8\% possuíam o pVHL, o que refletia em maior gravidade dos HBs, com recidiva tumoral local $(\mathrm{p}=0,027)$, como também foi mencionado por Koo et al. (2016). Sob outra perspectiva, os sintomas de pacientes com HBs-VHL ocorrem mais precocemente (30-40 anos) do que em pacientes com HBs esporádicos (40-50 anos).

No estudo de Wang et al. (2020), 187 pacientes possuíam HBs na medula espinhal e cerebelo, sendo 102 homens e 85 mulheres. Desses, 23\% foram diagnosticados com a Síndrome de von Hippel-Lindau. Além disso, foi visto que os pacientes > 60 anos obtiveram risco significamente menor de VHL e menor taxa de recorrência tumoral, como também pontuado por Westwick et al. (2016). No entanto, Wang et al. (2020) evidenciaram que o risco de possuir VHL em pacientes com HBs correlacionou-se negativamente com a idade do paciente e os pacientes do grupo de crianças/adolescentes apresentaram o maior risco de VHL. Dessa forma, pode-se inferir que a idade não influencia no risco de possuir HBs-VHL.

\section{Conclusão}

De acordo com as literaturas analisadas, conclui-se que há moderada prevalência da Síndrome de von Hippel-Lindau em pacientes portadores de hemangioblastomas do Sistema Nervoso Central. Dentre os estudos, a menor taxa de prevalência de HBs-VHL foi de $23 \%$, enquanto a maior taxa foi de aproximadamente $60 \%$, com uma média geral de $\pm 39,2 \%$, ao serem considerados todos os estudos que envolveram esses pacientes.

Sugere-se, portanto, o desenvolvimento de novos estudos restrospectivos e meta-análises a respeito da prevalência da Síndrome de von Hippel-Lindau em pacientes portadores de hemangioblastomas do Sistema Nervoso Central para que, dessa forma, a temática seja melhor elucidada, visto que a prevalência é muito variada em diferentes literaturas.

\section{Referências}

Ammerman, J. M., Lonser, R. R., Dambrosia, J., Butman, J. A., \& Oldfield, E. H. (2006). Long-term natural history of hemangioblastomas in patients with von Hippel-Lindau disease: implications for treatment. Journal of neurosurgery, 105(2), 248-255.

Cervio, A., Villalonga, J. F., Liñares, J. M., Mormandi, R., Condomí Alcorta, S., \& Salvat, J. (2015). Tratamiento quirúrgico de los hemangioblastomas del sistema nervioso central. Rev Argent Neuroc, 29(3), 117-131.

Cheng, J., Liu, W., Zhang, S., Lei, D., \& Hui, X. (2017). Clinical features and surgical outcomes in patients with cerebellopontine angle hemangioblastomas: retrospective series of 23 cases. World neurosurgery, 103, 248-256. 
Research, Society and Development, v. 11, n. 2, e44311226018, 2022

(CC BY 4.0) | ISSN 2525-3409 | DOI: http://dx.doi.org/10.33448/rsd-v11i2.26018

Chittiboina, P., \& Lonser, R. R. (2015). Doença de Von Hippel-Lindau. Manual de neurologia clínica, 132, 139-156. https://doi.org/10.1016/B978-0-444-62702$5.00010-\mathrm{X}$

Findeis-Hosey, J. J., McMahon, K. Q., \& Findeis, S. K. (2016). Von hippel-lindau disease. Journal of Pediatric Genetics, 5(02), 116-123.

Frantzen, C., Links, T. P., \& Giles, R. H. (2012). von Hippel-Lindau disease. GeneReviews. Seattle (WA): University of Washington, Seattle.

Guerrero-Galindo, R., Castrejón, E., Aguirre-Portillo, L., Cervantes-Michel, J. L., \& Espejo-Plascencia, I. (2012). Intramedullary hemangioblastoma. Archivos de Neurociencias, 17(4), 247-249.

Kanno, H., Kobayashi, N., \& Nakanowatari, S. (2014). Pathological and Clinical Features and Management of Central Nervous System Hemangioblastomas in von Hippel-Lindau Disease. Journal of kidney cancer and VHL, 1(4), 46-55. https://doi.org/10.15586/jkcvhl.2014.12

Koo, H. W., Park, J. E., Cha, J., Kim, D. J., Kang, S. G., Lim, S. C., \& Suh, D. C. (2016). Hemangioblastomas with leptomeningeal dissemination: Case series and review of the literature. Acta neurochirurgica, 158(6), 1169-1178.

Lonser, R. R., Glenn, G. M., Walther, M., Chew, E. Y., Libutti, S. K., Linehan, W. M., \& Oldfield, E. H. (2003). von Hippel-Lindau disease. The Lancet, 361(9374), 2059-2067.

Maher, E. R., Neumann, H. P., \& Richard, S. (2011). von Hippel-Lindau disease: A clinical and scientific review. European Journal of Human Genetics, 19(6), 617-623.

Malleo, G. (2010). Pancreatic cystic tumours: when to resect, when to observe. European review for medical and pharmacological sciences, 14, 395-406.

Nordstrom-O'Brien, M., van der Luijt, R. B., van Rooijen, E., van den Ouweland, A. M., Majoor-Krakauer, D. F., Lolkema, M. P., ... \& Giles, R. H. (2010). Genetic analysis of von Hippel-Lindau disease. Human mutation, 31(5), 521-537.

Patel, N. P., Robinson, T. M., Lesley, W. S., Garrett, D., Shan, Y., \& Huang, J. H. (2019). Hemangioblastoma Retromedular Imitando um Aneurisma da Artéria Cerebelar Inferior Posterior: Relato de Caso e Revisão de Literatura. Neurocirurgia Mundial, 122, 165-170. https://doi.org/10.1016/j.wneu.2018.10.188

Pavesi, G., Feletti, A., Berlucchi, S., Opocher, G., Martella, M., Murgia, A., \& Scienza, R. (2008). Neurosurgical treatment of von Hippel-Lindau-associated hemangioblastomas: benefits, risks and outcome. Journal of neurosurgical sciences, 52(2), 29-36.

Sampaio, D. B., Lima, W. P. de, Matias, I. de S., Saraiva, I. S., Ferreira, M. L., Ramos, V. M., \& Ribeiro, P. J. T. (2022). Treatment of hemangiomas in the head and neck region: integrative literature review. Research, Society and Development, 11(1), e43811125236. https://doi.org/10.33448/rsd-v11i1.25236

Seizinger, B. R., Rouleau, G. A., Ozelius, L. J., Lane, A. H., Farmer, G. E., Lamiell, J. M., ... \& Gusella, J. F. (1988). Von Hippel-Lindau disease maps to the region of chromosome 3 associated with renal cell carcinoma. Nature, 332(6161), 268-269.

Wanebo, J. E., Lonser, R. R., Glenn, G. M., \& Oldfield, E. H. (2003). The natural history of hemangioblastomas of the central nervous system in patients with von Hippel—Lindau disease. Journal of neurosurgery, 98(1), 82-94.

Wang, Q., Meng, S., Cheng, J., Zhang, S., Ju, Y., Fang, Y., ... \& Hui, X. (2020). Central nervous system hemangioblastomas: An age-stratified analysis. Clinical Neurology and Neurosurgery, 199, 106281.

Westwick, H. J., Giguère, J. F., \& Shamji, M. F. (2016). Incidence and prognosis of spinal hemangioblastoma: a surveillance epidemiology and end results study. Neuroepidemiology, 46(1), 14-23. 